\title{
Optically nonlinear chalcogenide glasses for all- optical signal processing
}

\author{
Barry Luther-Davies, Douglas Bulla, Rongping Wang, Amrita Prasad, Steve Madden, Duk-Yong Choi \\ Centre for Ultrahigh-bandwidth Devices for Optical Systems \\ Laser Physics Centre, Research School of Physics and Engineering \\ The Australian National University, Canberra ACT0200, Australia
}

\begin{abstract}
I discuss our work to optimize of the properties of highly nonlinear $\mathrm{Ge}_{\mathrm{x}} \mathrm{As}_{\mathrm{y}} \mathrm{Se}_{1-\mathrm{x}-\mathrm{y}}$ chalcogenide glasses for all-optical signal processing.
\end{abstract}

\section{INTRODUCTION}

Chalcogenide glasses are an important class of amorphous materials used as phase-change optical memories; as solar cells; and in photonics. They contain the chalcogen elements $\mathrm{S}$, Se and Te - covalently bonded to network formers such as As, Ge, Ga, Si, Sb. Being composed of weakly-bonded heavy elements leads to particular characteristics. These include broad transmittance in the mid-IR (beyond $20 \mu \mathrm{m}$ for Te-based glasses); optical gaps in the visible for near IR; high index of refraction (2-3); and relatively low glass transition temperatures. Weak chemical bonding also allows the structure of the glass network to be changed by exposure to energy in the form of heat, light, x-rays, etc. This leads to photo-structural phenomena such as photo-darkening and photo-crystallization which lends to applications, for example, as an x-ray photoresist or phase-change memories.

Applications in photonics have traditionally focussed on the production of bulk optical components for the mid-IR. However, there has been a growing interest in guided wave optics based on chalcogenides - optical fibers and waveguides. Chalcogenide optical fibers are attractive for transporting midIR light and for rare earth doping to create optical amplifiers and infra-red lasers. Our particular interest has focussed on their favourable nonlinear optical properties to achieve alloptical processing of high-speed telecommunications signals.

All-optical signal processing places some extreme requirements on the properties of the glass. Whilst obtaining high optical nonlinear response is paramount, simultaneously the devices must have negligible optical losses and be stable at the high fluences at $1550 \mathrm{~nm}\left(10-50 \mathrm{MW} / \mathrm{cm}^{2} \mathrm{CW}\right)$. Clearly the elimination of photo-structural effects is important if stable devices are to be obtained.

A challenge with the chalcogenides is that they form optically-transparent glasses over a very wide range of compositions and as a result it is difficult to predict a priori the best composition. Since our focus is ultimately on materials with the best third order nonlinear response, according to the semi-empirical Miller's rule [1] we should look for glasses with the highest refractive index. A more physical approach introduced by Sheik-Bahae et al. [2] suggests that glasses with optical gaps slightly exceeding twice the energy of the optical photons will have the largest nonlinearity but also negligible two-photon absorption.
Nevertheless, it is still difficult to predict which glass compositions to choose. An additional issue is that the properties of thin films required for waveguide devices are often very different from those of the equivalent bulk glass produced by melt-quenching. This arises because films are formed in non-equilibrium conditions and as a result can become trapped in an amorphous state different from the ideal glass. Films are often less stable compared with bulk materials.

Against this background, we set out to identify compositionproperty relationships for glasses in the $\mathrm{Ge}_{\mathrm{x}} \mathrm{As}_{\mathrm{y}} \mathrm{Se}_{1-\mathrm{x}-\mathrm{y}}$ system with the aim of obtaining a better understanding of the factors leading to nonlinear thin films suitable for all-optical processing. Our choice of this particular glass system was motivated by the fact that there has been a lot of research into this system, and the range of glass-forming compositions is large making it an ideal candidate for such studies.

\section{EXPERIMENTS}

Our presentation is guided by the ideas of Phillips [3], Thorpe [4] and Tanaka [5] who used the mean coordination number $(\mathrm{MCN})$ - the sum of the products the covalent coordination number times the abundance of the constituent atoms - to parameterize chalcogenide glasses with widely varying compositions. We fabricated $\mathrm{Ge}_{\mathrm{x}} \mathrm{As}_{\mathrm{y}} \mathrm{Se}_{1-\mathrm{x}-\mathrm{y}}$ glasses with MCN between 2.2 and 2.94 using the standard meltquenching method. It is worth noting that chemically stoichiometric glasses are restricted to $\mathrm{MCN}=2.4-2.67$. When $\mathrm{MCN}<2.4$ the glasses are Se rich whilst for $\mathrm{MCN}>2.67$ they must be Se-poor. According to Phillips [3] and Thorpe [4] at $\mathrm{MCN}=2.4$ the glass undergoes an important transition from a floppy to rigid network whilst at $\mathrm{MCN}=2.67$ Tanaka [5] found a second transition from the rigid to a stressed-rigid phase. They predicted that the physical properties of the glass should reflect these fundamental changes in the network topology.

At the beginning of this study we wanted to answer a series of questions. We wanted to find our how parameters such as the linear and nonlinear index of refraction; the optical losses; the energy gap; the glass transition temperature; etc., varied with MCN. Would such data provide guidance as to which glasses would have the best properties and particularly the best stability for all-optical processing? We were also interested in creating films using thermal evaporation and determining how their properties differed from those of their parent glasses.

In order to elucidate such behaviour a wide range of measurements were made. These included measurements of glass density and index of refraction; z-scans to determine the 
third order nonlinearity; measurements of the Tauc gap using thin glass samples; EDX and Laser-Ablation Inductively Coupled Plasma Mass Spectrometry (LA-ICP-MS) to check chemical composition of both films and bulk glasses; Raman spectroscopy and XPS to analyse chemical bonding; laser ultrasonic pulse interferometry to determine elastic moduli; and XRD to check for amorphicity; etc.

Measurements were carried out on the glasses produced by melt-quenching as well as thin films produced by thermal evaporation under high vacuum $\left(\approx 10^{-7}\right.$ torr $)$ from a temperature controlled baffled boat.

\section{RESULTS}

One of the most striking conclusions from our data is that a strong correlation indeed exists between many physical properties and MCN. The glass density; refractive index; elastic moduli; and Tauc gap all show abrupt changes in their MCN-dependence at both $\mathrm{MCN}=2.4$ and 2.67 [6,7]. An example is shown in Fig. 1 where we plot the refractive index of both thermally evaporated films and bulk glass as a function of MCN. Since the index of refraction is related to both glass density and Tauc gap it is perhaps not surprising that these parameters also display analogous changes as do the elastic moduli as shown in Fig. 2. Similar transitions could be found in Raman data where bands associated with Se-Se bonds disappear for $\mathrm{MCN} \approx 2.4-2.5$ and new bands associated with defect bonds appear for $\mathrm{MCN}>2.7$.

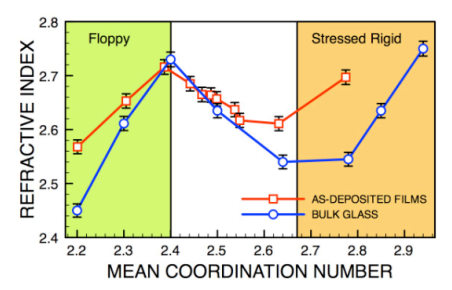

Fig. 1. Refractive index of bulk and thin films of $\mathrm{Ge}_{x} \mathrm{As}_{\mathrm{y}} \mathrm{Se}_{1-\mathrm{x}-\mathrm{y}}$ glass plotted as a function of mean coordination number. The floppy and stressed rigid networks are indicated by the shading.

On the basis of both Miller's rule (and the Sheik-Bahae model [2]), one would expect that the highest nonlinear refractive index would, therefore, occur at $\mathrm{MCN} \approx 2.4$ and $\mathrm{MCN}>2.8$ which corresponds the highest linear indices and smallest Tauc gaps. This was confirmed by the z-scan measurements with the maximum nonlinearity measured to be around 400 times that of fused silica.

The question arises, therefore, is, if the nonlinearity is the same, which are the better glasses for nonlinear waveguides? Since the glass transition temperature increases monotonically with MCN therefore one might imagine the high MCN glasses could be more stable. However, high MCN glasses contain large numbers of defect bonds and our loss measurements (and Raman data) show that the type of defects present in Se-poor glasses cause high optical loss. Therefore, in spite of their lower $\mathrm{T}_{\mathrm{g}}$, glasses with MCN in the 2.4-2.5 range offer a better combination of properties: linear and nonlinear.
However the most striking advantage of intermediate MCN glasses emerges from studies of the properties of evaporated thin films. A clue to this appears in Fig. 1 that compares the refractive index of as-deposited films with the bulk glass ${ }^{7}$. Clearly, high and low MCN films have substantially different refractive index values from the bulk whilst for MCN from about 2.4-2.5 the differences are very small. What does this imply? By using XPS to probe the nature of the bonds we found that films in this intermediate range formed with chemical bonds indistinguishable from the bulk, whereas outside that range the chemical bonds were quite different. Annealing studies showed that films with these intermediate MCNs were completely stable whereas at high or low MCN annealing caused changes in the chemical bonds and a large changes in other physical properties.

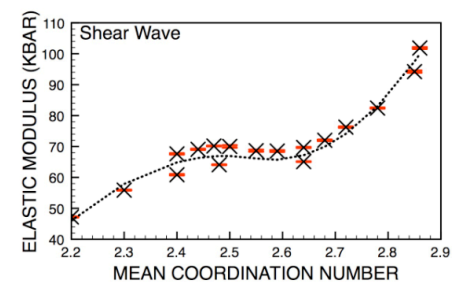

Fig. 2. Measurement of shear wave elastic moduli as a function of MCN.

We suggest this behaviour can be explained in terms of the energy landscape discussed by Lucas [8] and reflects a difference in the topology of the potential hypersurface. Around $\mathrm{MCN}=2.45$, the potential hypersurface contains a broad well-defined minimum representative of the ideal glass. As a result the films have a strong tendency to form with the same bond configuration as the glass even at room temperature. For high or low MCN, the potential hypersurface is more complex, with significant energy barriers separating the different possible amorphous networks from the ideal glass. As-deposited films get trapped by the barriers and can only relax towards the ideal glass when heated. Glasses with $\mathrm{MCN} \approx 2.45$ are therefore fundamentally more stable - a desirable property for all-optical devices.

\section{ACKNOWLEDGEMENTS}

The authors gratefully acknowledge the support of the Australian Research Council for this work.

\section{REFERENCES}

[1] C.C Wang, Phys. Rev. B 2, 2048 (1970).

[2] M. Shiek-Bahae, D. C. Hutchings, D. J. Hagan and E. W. Van Stryland, IEEE J. Quantum. Electron. 27, 1296-1309 (1991).

[3] J.C.Phillips, J.Non-Cryst.Solids 34, 153(1979).

[4] M.F.Thorpe, J.Non-Cryst.Solids 57, 355(1983); M.F.Thorpe, D.J.Jacobs, M.V.Chubynsky, and J.C.Phillips, J.Non-Cryst.Solids 266-269, 859(2000).

[5] K. Tanaka, Phys. Rev. B 39, 1270 (1989)

[6] R.P. Wang, A. Smith, B. Luther-Davies, H. Kokkonen, I. Jackson, J. Appl. Phys. 105, 056109 (2009).

[7] D.A.P. Bulla, R.P. Wang, A. Prasad, A.V. Rode, S.J. Madden, B. LutherDavies, Appl. Phys.A 96, 615 (2009).

[8] P. Lucas, J. Phys.: Condens, Matter 18, 5629 (2006). 


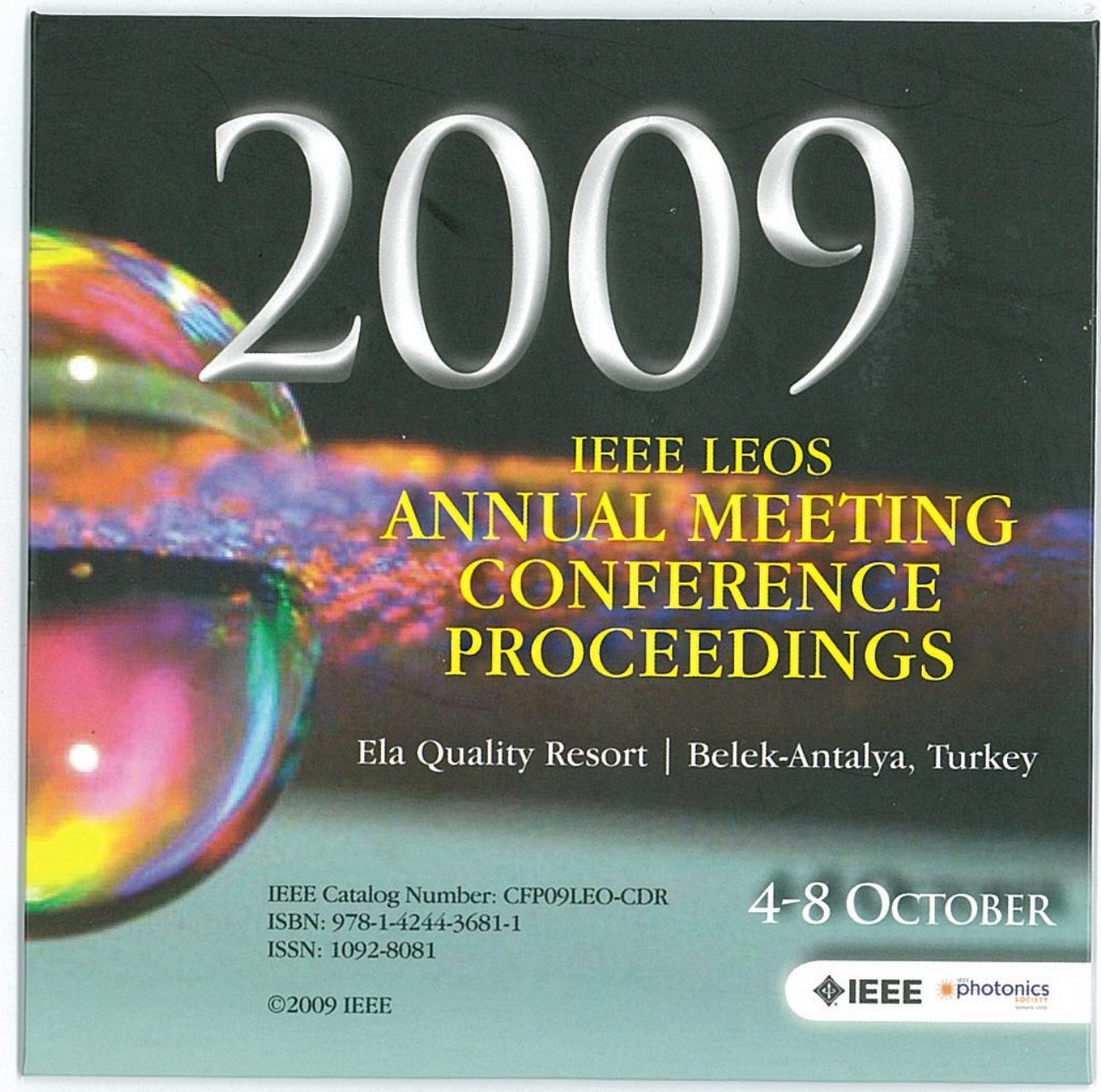

